\title{
Process analytics in science and industry
}

\author{
Rudolf W. Kessler ${ }^{1}$
}

Published online: 29 November 2016

(C) Springer-Verlag Berlin Heidelberg 2016

Process analytical technology (PAT) has attracted enormous interest in recent years. The US Food and Drug Administration's PAT and quality by design platform more than 10 years ago and recently the German initiative for the fourth industrial revolution "Industry 4.0," the US Industrial Internet Consortium, the Internet of things, and the Chinese strategy "Made in China 2025" are just a few examples. The role of PAT in all these concepts is essentially to promote an understanding of the process at the basic mechanistic level by introducing in-line smart sensors; thus enabling knowledge-based production in the future.

Cost pressure, globalization, and quality assurance continue to be challenges and will undoubtedly stimulate significant further demands for PAT automation in all industries. The future activities of the chemical industry will focus on knowledge-based production at all levels. The pharmaceutical industry is trying to change from a more reactive production to a more proactive production. Because of the complexity of biotechnological operations and complexity in medical applications, multimodularity of sensors is essential. Finally, the food and animal feed industry recognizes the importance of automation. Tracking and tracing will enhance food safety and result in less food spoilage. New monitoring equipment and smart controls

Published in the topical collection Process Analytics in Science and Industry with guest editor Rudolf W. Kessler.

Rudolf W. Kessler

rudolf.kessler@ reutlingen-university.de

1 STZ Prozesskontrolle und Datenanalyse, Kaiserstr. 66, 72764 Reutlingen, Germany have to be introduced in business areas where increased productivity and sustainability are the overall key factors. PAT and especially spectroscopy are essential to provide context-sensitive information on a molecular level for smart monitoring and control of production in the processing and manufacturing industries.

The articles in this topical collection of Analytical and Bioanalytical Chemistry describe a great variety of the abovementioned concepts and illustrate how PAT can be introduced in different application areas and industries. Applications include examples from the chemical and pharmaceutical industries, from biochemical production, and from the food and feed industry and also new concepts in biomedical use of instrumentation. The development of new analytical equipment is also shown, along with the importance of the search for smart methods for calibration and data analysis and new strategies for system integration to control complex unit operations. The articles also provide broad insight into the aspects of process analytics as a truly transdisciplinary tool. Process chemists, process engineers, chemometricians, and many other technologists must work together, and this is demonstrated perfectly in the research articles. A goal is to increase confidence in this complex technology. This is a task that researchers, equipment manufacturers, and users will have to address together.

"The future of industrial automation will be arbitrarily modifiable and expandable (flexible), connect arbitrary components of multiple producers (networked), enabling its components to perform tasks related to its context independently (self-organizational) and emphasizes ease of use (user-oriented)." This is the statement to describe the fourth industrial revolution, or the Internet of things [1, 2].

It is important to emphasize that the transdisciplinary nature of PAT, as mentioned before, is a key issue to 
successfully introduce smart sensors in industry and to bring different cultures together. Thus, analytical chemistry must play a major role in educational institutions to provide the breadth that is necessary for technologies that involve multiple disciplines. As guest editor, I hope that this topical collection will provide an overview of current research into process analytics in science and industry.

\section{References}

1. Meixner G, Petersen N, Koessling H. User Interaction Evolution in the SmartFactory ${ }^{\mathrm{KL}}$. In: Proceedings of the 24th BCS Interaction Specialist Group Conference, 211-220, Dundee, United Kingdom - September 06 - 10, 2010. 2010.

2. Kessler RW. Perspectives in process analysis. J Chemometrics. 2013;27:369-78. doi:10.1002/cem.2549.

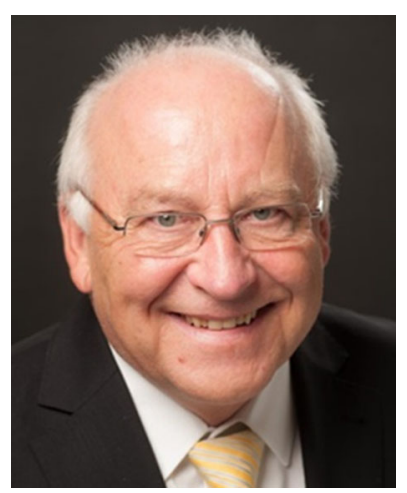

Rudolf W. Kessler is Professor Emeritus of Chemistry at Reutlingen University, Germany. After his undergraduate studies in chemistry and his $\mathrm{PhD}$ studies in physical chemistry and spectroscopy, he worked for some years at Mercedes-Benz in Stuttgart, Germany. His research interests are in the field of knowledgebased production, spectral imaging, and also near-field spectroscopy, and he has published a book on process analysis. He is the head of a Steinbeis Center for Technology Transfer for process control and data analysis with a focus on consulting in the area of process analytical technology (PAT) and quality by design in industry. During his career, he has established, together with his colleagues, a strong PAT network in Germany and Europe, and for many years he was a member of the board of the PAT Working Group of the German Chemical Society (GDCh) and DECHEMA. He is also one of the initiators and organizers of the EuroPact conferences. 